\title{
Facilitation of Corticospinal Excitability According to Motor Imagery and Mirror Therapy in Healthy Subjects and Stroke Patients
}

\author{
Youn Joo Kang, M.D., Jeonghun Ku, Ph.D. ', Hyun Jung Kim, M.D., Hae Kyung Park, M.D. \\ Department of Rehabilitation Medicine, Eulji Hospital, Eulji University School of Medicine, Seoul 139-711, \\ ${ }^{1}$ Department of Biomedical Engineering, Keimyung University, Daegu 704-701, Korea
}

Objective To delineate the changes in corticospinal excitability when individuals are asked to exercise their hand using observation, motor imagery, voluntary exercise, and exercise with a mirror.

Method The participants consisted of 30 healthy subjects and 30 stroke patients. In healthy subjects, the amplitudes and latencies of motor evoked potential (MEP) were obtained using seven conditions: (A) rest; (B) imagery; (C) observation and imagery of the hand activity of other individuals; (D) observation and imagery of own ipsilateral hand activity; (E) observation and imagery of the hand activity of another individual with a mirror; (F) observation and imagery of own symmetric ipsilateral hand activity (thumb abduction) with a mirror; and (G) observation and imagery of own asymmetric ipsilateral hand activity (little finger abduction) with a mirror. In stroke patients, MEPs were obtained in the A, C, D, E, F conditions.

Results In both groups, increment of the percentage MEP amplitude (at rest) and latency decrement of MEPs were significantly higher during the observation of the activity of the hand of another individual with a mirror and during symmetric ipsilateral hand activity on their own hand with a mirror than they were without a mirror. In healthy subjects, the increment of percentage MEP amplitude and latency decrement were significantly higher during the observation of the symmetric ipsilateral hand activity with a mirror compared to the observation of the activity of the asymmetric ipsilateral hand with a mirror of their own hand.

Conclusion In both groups, corticospinal excitability was facilitated by viewing the mirror image of the activity of the ipsilateral hand. These findings provide neurophysiological evidence supporting the application of various mirror imagery programs during stroke rehabilitation.

Key Words Stroke, Corticospinal excitability, Transcranial magnetic stimulation, Motor imagery, Mirror therapy

Received January 12, 2011; Accepted October 5, 2011

Corresponding author: Hae Kyung Park

Department of Rehabilitation, Eulji Hospital, 280-1, Hagye dong, Nowongu, Seoul 139-711, Korea

Tel: +82-2-970-8315, Fax: +82-2-979-8268, E-mail: md52516@hanmail. net

(c) This is an open-access article distributed under the terms of the Creative Commons Attribution Non-Commercial License (http:// creativecommons.org/licenses/by-nc/3.0) which permits unrestricted noncommercial use, distribution, and reproduction in any medium, provided the original work is properly cited.

Copyright $\odot 2011$ by Korean Academy of Rehabilitation Medicine

\section{INTRODUCTION}

The incidence of stroke is growing, and more than $50 \%$ of stroke patients suffer from disabilities in the upper extremities that hinder daily living. ${ }^{1}$ In addition, more than $50 \%$ of stroke patients report continuous disability of upper extremity function after conventional treatment, and learned nonuse of the injured arm is fre- 
quently observed. ${ }^{2}$ For these reasons, programs aimed at restoring the function of upper extremities are an important part of stroke rehabilitation.

The conventional rehabilitative method emphasizes and focuses on passive facilitation and training compensation to achieve independence of daily activities. These include palpation and sensory stimulation (developed by Rood in the 1950s), proprioceptive neuromuscular facilitation (developed in the 1960s), and exercise treatment (developed by Brunnstrom in 1970). Recently, new treatment methods for upper extremity rehabilitation based on the motor learning theory have been assessed. According to the motor learning theory, many important factors should be considered for successful treatment, such as repetition, focusing, task variability, stimulation of various sensory motor stimuli, and feedback. ${ }^{3}$ Representative treatment methods that have been emerging recently include the constraint-induced movement theory, robot-arm training, training using virtual environment technology (VET), motor imagery, and mirror therapy. ${ }^{4}$ Even though results supporting the effectiveness of these methods are scarce, a wide range of randomized controlled clinical trials (RCTs) have been conducted. ${ }^{5}$

According to previous study, only observations of the skilled movement of other individuals and continuous motor imagery were effective in motor training. ${ }^{6}$ This relationship between motor imagery and real exercise could be explained by the transformation-process that the perception and cognition of the movements to the physical movements. Recent neuroimaging and transcranial magnetic stimulation (TMS) studies showed that the primary motor area (M1) was activated by the observation and imagery of movement, ${ }^{7,8}$ and that the motor imagery elicited stronger motor evoked potentials (MEPs) when the imagined movements were complex than simple movement. ${ }^{8}$ Many studies showed that complex and task-specific kinesthetically congruent imagery facilitates the brain more than simple visual imagery and observation does. ${ }^{9,10}$ Several experimental studies suggest that various imagined action and motor imagery programs enhance motor recovery of hemiplegic patients, as assessed through RCTs. ${ }^{6,11,12}$

Mirror therapy is effective for the treatment of phantom limb pain in amputee patients, ${ }^{13}$ so many studies have applied this therapy while treating hemiplegic patients to investigate and validate its clinical effects based on the hypothesis that the visual illusion evoked by the mirror reflection of the unaffected arm, while simultaneously blocking the visualization of the affected arm, would improve the motor abilities of these patients. ${ }^{14,15}$ The exercise therapy paradigm uses a mirror that can be easily installed and used in any place, thus providing patients with an opportunity to practice repeatedly. However, some disadvantages of this method have been displayed based on the reduced clinical compliance for stroke patients. Further, there is insufficient theoretical evidence that suggests that visual illusion can provoke facilitation of cortical excitability in the affected hemisphere after a stroke.

After a unilateral stroke, severe damage may modify transcallosal inhibition resulting in decreased activity in the ipsilesional motor cortex and hyperexcitability of the contralesional motor cortex. ${ }^{16,17}$ Abnormally strong interhemispheric inhibition from the contralateral hemisphere leads to poor functional outcome during the recovery phase of a stroke and ipsilesional cortical activation is important for good motor recovery. ${ }^{18}$ Therefore, priming of the ipsilesional motor cortex in the acute recovery period results in enhanced ipsilesional lateralization. This return to balanced corticomotor excitability between the two hemispheres is a very important strategy in stroke rehabilitation. ${ }^{19}$

We hypothesized that motor imagery, observation, voluntary exercise, and mirror therapy modulate ipsilesional corticospinal excitability both in stroke patients and in healthy subjects. To the best of our knowledge, studies aimed at investigating brain corticospinal activation in response to observation, imagery, and mirror therapy were performed using healthy populations exclusively; in addition, these studies yielded inconsistent results. ${ }^{20,21}$ Therefore, in this study, MEPs elicited by the observation of movements, imagery, voluntary exercise, and the mirror paradigm were investigated in stroke patients and in a healthy population. For this purpose, we used single-pulse TMS, and the amplitude and latency of MEPs were derived from the target muscle. TMS was beneficial in identifying differences in corticospinal excitability according to various experimental conditions using a real-time analysis. ${ }^{22}$ 


\section{MATERIALS AND METHODS}

\section{Participants}

For this experiment, 30 right-handed, healthy subjects (18 men and 12 women) and 30 stroke patients (18 men and 12 women) were recruited. The mean age of the two groups was $27.97 \pm 2.44$ years in the healthy subjects and
$66.03 \pm 11.03$ years in the stroke patients. The healthy volunteers had no history of neurological disease, and no abnormalities were observed in physical, neurological, or musculoskeletal examinations. The stroke patients (1) had suffered a primary ischemic or hemorrhagic stroke as revealed by computed tomography (CT) or magnetic resonance imaging (MRI) scans, (2) presented

Table 1. Demographic and Clinical Characteristics of 30 Stroke Patients

\begin{tabular}{|c|c|c|c|c|c|c|}
\hline Patients & Sex & $\begin{array}{c}\text { Age } \\
\text { (years) }\end{array}$ & $\begin{array}{c}\text { Weeks } \\
\text { since onset }\end{array}$ & Etiology & Site of lesion & $\begin{array}{c}\text { Function } \\
\text { independence } \\
\text { measure }\end{array}$ \\
\hline 1 & M & 78 & 5 & Infarction & Lt. Lat. medulla & 88 \\
\hline 2 & M & 69 & 6 & Infarction & Lt. BG, Th (subcortical) & 94 \\
\hline 3 & M & 55 & 5 & Infarction & Rt. BG (subcortical) & 90 \\
\hline 4 & $\mathrm{~F}$ & 55 & 10 & Infarction & Lt. BG, Th (subcortical) & 94 \\
\hline 5 & $\mathrm{~F}$ & 68 & 6 & Infarction & Lt. F, P (cortical and subcortical) & 92 \\
\hline 6 & M & 75 & 5 & Infarction & Rt. corona radiata & 94 \\
\hline 7 & M & 69 & 7 & Hemorrhage & Rt. F, P, O (cortical and subcortical) & 92 \\
\hline 8 & $\mathrm{~F}$ & 57 & 8 & Hemorrhage & Rt. pontine & 94 \\
\hline 9 & M & 72 & 6 & Infarction & Lt. BG, Th (subcortical) & 92 \\
\hline 10 & $\mathrm{~F}$ & 65 & 8 & Infarction & Rt. BG (subcortical) & 92 \\
\hline 11 & M & 71 & 5 & Infarction & Rt. BG, Th (subcortical) & 90 \\
\hline 12 & M & 72 & 6 & Infarction & Rt. Lat. medulla & 94 \\
\hline 13 & M & 75 & 8 & Infarction & Rt. BG (subcortical) & 94 \\
\hline 14 & $\mathrm{~F}$ & 67 & 10 & Infarction & Rt. BG (subcortical) & 94 \\
\hline 15 & M & 67 & 7 & Infarction & Rt. BG (subcortical) & 92 \\
\hline 16 & M & 38 & 4 & Hemorrhage & Rt. BG (subcortical) & 90 \\
\hline 17 & M & 52 & 6 & Hemorrhage & Lt. F (cortical and subcortical) & 94 \\
\hline 18 & M & 69 & 5 & Infarction & Rt. pontine & 88 \\
\hline 19 & $\mathrm{~F}$ & 80 & 3 & Infarction & Lt. BG, Th (subcortical) & 88 \\
\hline 20 & M & 64 & 6 & Hemorrhage & Lt. F, T, P (cortical and subcortical) & 94 \\
\hline 21 & $\mathrm{~F}$ & 61 & 4 & Infarction & Lt. IC (subcortical) & 90 \\
\hline 22 & $\mathrm{~F}$ & 89 & 5 & Infarction & Lt. pontine & 88 \\
\hline 23 & M & 41 & 4 & Infarction & Lt. Lat medullary & 90 \\
\hline 24 & M & 58 & 5 & Infarction & Lt. corona radiata & 94 \\
\hline 25 & M & 76 & 5 & Infarction & Lt. BG (subcortical) & 88 \\
\hline 26 & M & 59 & 3 & Infarction & Lt. BG (subcortical) & 88 \\
\hline 27 & M & 69 & 7 & Infarction & Rt. pontine & 90 \\
\hline 28 & $\mathrm{~F}$ & 65 & 7 & Hemorrhage & Lt. cerebellum & 94 \\
\hline 29 & $\mathrm{~F}$ & 77 & 6 & Infarction & Lt. BG (subcortical) & 88 \\
\hline 30 & M & 74 & 9 & Hemorrhage & Lt. $\mathrm{P}, \mathrm{O}$ (cortical and subcortical) & 94 \\
\hline
\end{tabular}

Rt: Right, Lt: Left, BG: Basal ganglia, Th: Thalamus, F: Frontal lobe, P: Parietal lobe, O: Occipital lobe 
mild to moderate paresis of the upper extremity (upper extremity manual muscle function test $>$ grade 3 ), (3) lacked any additional neurological disease causing a motor deficit, and (4) showed no severe deficits in communication, memory, or understanding, with a score $>24$ in the Mini-Mental State Examination. The causes of stroke were infarction in 23 patients and hemorrhage in seven patients; 13 patients had a right-side lesion and 17 patients had a left-side lesion (Table 1). Participants that had a pacemaker, a contraindication to magnetic stimulation (such as a history of seizure), severe depression, apraxia, possibility of pregnancy, or were pregnant were excluded. All participants that consented to participate in this study were informed about TMS and the experimental protocol, both of which were approved by the institutional review board of our hospital.

\section{Experimental design}

Experiment I: facilitation of corticospinal excitability during mirror therapy: Magnetic stimulation was achieved using the figure-eight coil by MagVenture ${ }^{\circledR}$ MagPro (MagVenture, Lucernemarken, Denmark) and electromyography (EMG) signals were measured using the EMG system AlpinebioMed ${ }^{\circledR}$ Keypoint (Fountain Valley, CA, USA). To localize the target for stimulation, each subject wore a hat marked with $3 \mathrm{~cm}$ squares; the center of the hat was positioned to the bisection line of the nasion and inion/ear-to-ear $(\mathrm{Cz})$ while sitting comfortably with both hands placed on a table. TMS was applied to the nondominant (right) hemisphere in healthy subjects and to the affected hemisphere in stroke patients. The electrodes used to measure MEPs in healthy subjects were attached to the motor point of the abductor pollicis brevis (APB) of the nondominant (left) hand for the active recording, and to the tendon of the corresponding muscle for reference (Fig. 1). By contrast, in stroke patients, MEPs were acquired from the same muscle that was assessed on the affected arm. The abduction of the thumb and fifth finger was performed at a constant pace of one time/second (s). The hot spot of the hand motor area was determined as the stimulation strength that yielded a hand movement for the first time while stimulating the motor area in the nondominant hemisphere for healthy subjects and the affected hemisphere for stroke patients. The motor threshold was defined as the lowest stimulus intensity sufficient to elicit
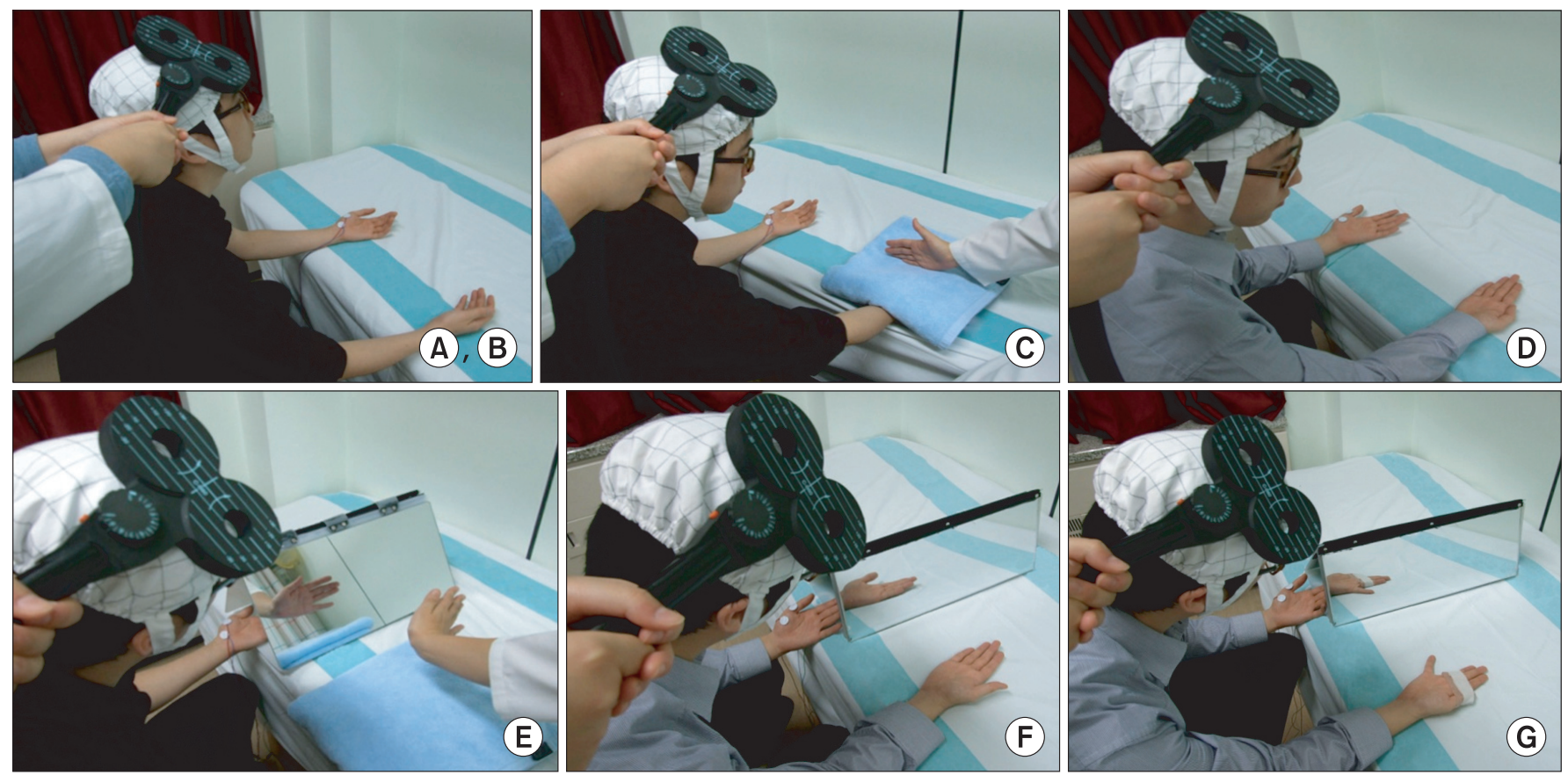

Fig. 1. (A, B) Relaxation and kinesthetic motor imagery state. (C) Observation and imagery of the activity of the hand of another individual without a mirror. (D) Observation and imagery of own hand activity without a mirror. (E) Observation and imagery of the activity of the hand of another individual with a mirror. (F) Observation and imagery of own symmetric hand activity with a mirror. (G) Observation and imagery of own asymmetric hand activity with a mirror. 
six MEPs of $>50 \mu \mathrm{V}$ in a series of ten stimuli delivered at intervals of at least $5 \mathrm{s.}^{23}$ Stimulus intensity was adjusted at $120 \%$ of motor threshold.

In the first experiment, the latency and the amplitude of the MEPs were examined in the resting state. The motor imagery, the observation and imagery of the activity of the hand of another individual (with or without a mirror), and the voluntary ipsilateral hand activity conditions (with or without mirror) were assessed in 30 healthy subjects and 30 stroke patients. All trials were randomly assigned and the mean latency and amplitude of twelve trials were obtained. During the recording, the nondominant hand (in healthy subject and affected hand in stroke patients) with the attached recording electrode was completely relaxed and any trial in which muscle contraction was observed in the EMG signal was excluded from the analysis. The following experiment was conducted in a random order, to eliminate the order effect, and a three minute rest was introduced between the tasks.

For healthy subjects: (A) Relaxation state (control): Subjects were completely relaxed and stimulated twelve times with TMS. The mean MEP latency and amplitude of twelve trials were obtained.

(B) Imagery: Subjects were asked to sit in a relaxed position and to imagine the abduction of their thumb.

(C) Observation and imagery of the activity of the hand of another individual without a mirror: Subjects were asked to imagine the abduction of their thumb while observing thumb abduction in another individual (experimenter).

(D) Observation and imagery of own ipsilateral hand activity without a mirror: Subjects were asked to imagine the abduction of their thumb while observing the abduction of their right thumb. The abduction of the thumb was performed at a constant pace of one time/s.

(E) Observation and imagery of the activity of the hand of another individual with a mirror: Subjects observed the reflected image of the movement of another individual (experimenter). MEPs were assessed on the APB of the left thumb while observing the mirror image of a thumb abduction of another individual.

(F) Observation and imagery of own ipsilateral hand activity with a mirror: Subjects were asked to place their left arm in the mirror box, which was placed in the middle of the table, and to place their right arm to be reflected in the mirror. Subjects began to perform right thumb abduction and imagine movements similar to the reflected image observed on the mirror box. MEPs were assessed in the APB of the left thumb while observing the mirror image of voluntary ipsilateral thumb abduction.

(G) Observation and imagery of own ipsilateral asymmetric hand activity with a mirror (observationexecution mismatching): Subjects were asked to imagine asymmetric hand activities from the reflected mirror image of their own abduction of the right fifth finger. The placement of the arms and of the mirror box was the same as in the (F) condition. Four fingers (from the first to the fourth finger) were tied with paper tape to prevent any activity of the other fingers during the activity of the fifth finger. Subjects then performed abduction of the right fifth finger. MEPs were assessed in the APB of the left thumb while observing the asymmetric mirror image of the abduction of the right fifth finger (Fig. 1-G).

For stroke patients: (A) Relaxation state (control): Subjects were completely relaxed and stimulated twelve times with TMS. The mean MEP latency and amplitude of twelve trials were obtained.

(B) Observation and imagery of the activity of the hand of another individual without a mirror: Subjects were asked to imagine the abduction of their thumb while observing the abduction of the thumb of another individual (experimenter).

(C) Observation and imagery of own ipsilateral hand activity without a mirror: Subjects were asked to imagine the abduction of their thumb while observing the abduction of their unaffected ipsilateral thumb.

(D) Observation and imagery of the activity of the hand of another individual with a mirror: Subjects were asked to observe the mirror-reflected image of the movements of another individual (experimenter). MEPs were assessed in the APB of the affected thumb in the mirror box while observing the mirror image of a thumb abduction of another individual.

(E) Observation and imagery of own unaffected ipsilateral hand activity with a mirror: Subjects were asked to observe the mirror-reflected image of their own movements. Subjects began to perform abduction of the unaffected thumb and to imagine movements similar to the reflected image observed on the mirror box. MEPs were assessed in the APB of the affected thumb in the mirror box while observing the mirror image of their own 
unaffected thumb abduction.

Data analysis: The peak-to-peak amplitude and latency of the MEPs recorded in each condition were measured and averaged to derive mean values. The value of MEPs were not normally distributed, so individual mean values were transformed into a percentage of the MEP at rest for each subject. The individual percentage MEPs were then entered into a repeated measures one-way analysis of variance (ANOVA). The latency measurement was normally distributed and required no transformation. The Bonferroni multiple comparisons test was also used to compare conditions that were significantly different from the baseline $(\mathrm{p}<0.05)$. Independent sample T-test was used to compare mean MEP amplitudes and latencies between healthy subjects and stroke patients. All data were analyzed using the SPSS software package, version 12.

\section{RESULTS}

The comparison of the mean MEP amplitudes across the five testing conditions (A, C, D, E, F), revealed a pattern of significant differences between healthy subjects and stroke patients (Table 2, 3). The MEP amplitudes were higher in healthy subjects than in stroke patients $(p<0.001)$. However, the mean MEP latencies across the five testing conditions didn't show any differences between the two groups (Table 2, 3).

Facilitation of corticospinal excitability during motor imagery and mirror therapy in healthy subjects In 30 healthy subjects, the comparison of the percentage of MEP amplitudes across the six testing conditions revealed a pattern of significant differences during experimentation $\left(\mathrm{F}_{5,135}=282.6\right)(\mathrm{p}<0.001)$ (Fig. 2). The percentage of MEP amplitudes were higher in con-

Table 2. Amplitude and Latency of MEPs in Healthy Subjects

\begin{tabular}{lcc}
\hline & $\begin{array}{c}\text { Amplitude } \\
(\mathbf{m V})\end{array}$ & $\begin{array}{c}\text { Latency } \\
(\mathbf{m s e c})\end{array}$ \\
\hline A) Relaxation state (control) & $1.32 \pm 0.21$ & $21.56 \pm 0.75$ \\
\hline B) Imagery & $1.31 \pm 0.19$ & $20.77 \pm 0.56$ \\
\hline $\begin{array}{l}\text { C) Observation and imagery of the activity of the hand of another } \\
\text { individual without a mirror }\end{array}$ & $3.25 \pm 0.69$ & $19.78 \pm 0.66$ \\
\hline D) Observation and imagery of own hand activity without a mirror & $5.48 \pm 0.56$ & $16.99 \pm 0.71$ \\
\hline $\begin{array}{l}\text { E) Observation and imagery of the activity of the hand of another } \\
\text { individual with a mirror }\end{array}$ & $5.44 \pm 0.52$ & $18.52 \pm 0.68$ \\
\hline F) Observation and imagery of own hand activity with a mirror & $5.83 \pm 0.41$ & $16.49 \pm 0.58$ \\
\hline $\begin{array}{l}\text { G) Observation and imagery of own asymmetric hand activity with a mirror } \\
\text { (observation-execution mismatching) }\end{array}$ & $2.43 \pm 0.27$ & $20.42 \pm 0.71$ \\
\hline
\end{tabular}

Values are mean \pm SD

Table 3. Amplitude and Latency of MEPs in Stroke Patients

\begin{tabular}{lcc}
\hline & $\begin{array}{c}\text { Amplitude } \\
(\mathbf{m V})\end{array}$ & $\begin{array}{c}\text { Latency } \\
(\mathbf{m s e c})\end{array}$ \\
\hline A) Rest & $0.86 \pm 0.51$ & $21.47 \pm 2.87$ \\
$\begin{array}{l}\text { C) Observation and imagery of the activity of the hand of another } \\
\text { individual without a mirror }\end{array}$ & $0.89 \pm 0.51$ & $19.64 \pm 2.74$ \\
\hline D) Observation and imagery of own hand activity without a mirror & $1.19 \pm 0.78$ & $16.98 \pm 2.89$ \\
\hline $\begin{array}{l}\text { E) Observation and imagery of the activity of the hand of another } \\
\text { individual with a mirror }\end{array}$ & $1.05 \pm 0.67$ & $17.96 \pm 2.56$ \\
\hline F) Observation and imagery of own hand activity with a mirror & $1.26 \pm 0.79$ & $16.04 \pm 2.86$ \\
\hline
\end{tabular}

Values are mean \pm SD 
dition (C) observation and imagery than in condition (B) imagery only ( $\mathrm{p}<0.01$ ) (Fig. 2). Comparison of conditions (C) and (D) revealed that the percentage of MEP amplitude of (D) observation and imagery of own hand activity was higher than that of (C) observation and imagery of the activity of the hand of another individual $(p<0.001)$. The results of testing conditions using a mirror showed that the percentage of MEP amplitude was higher in the mirror condition of (E) observation and imagery of the activity of the hand of another individual with a mirror than in (C) observation and imagery of the activity of the hand of another individual without a mirror $(\mathrm{p}$ $<0.01$ ) (Fig. 2). The percentage of MEP amplitude was also higher in condition (F) observation and imagery of own hand activity with a mirror than in (D) observation and imagery of own hand activity without a mirror $(p<0.05)$

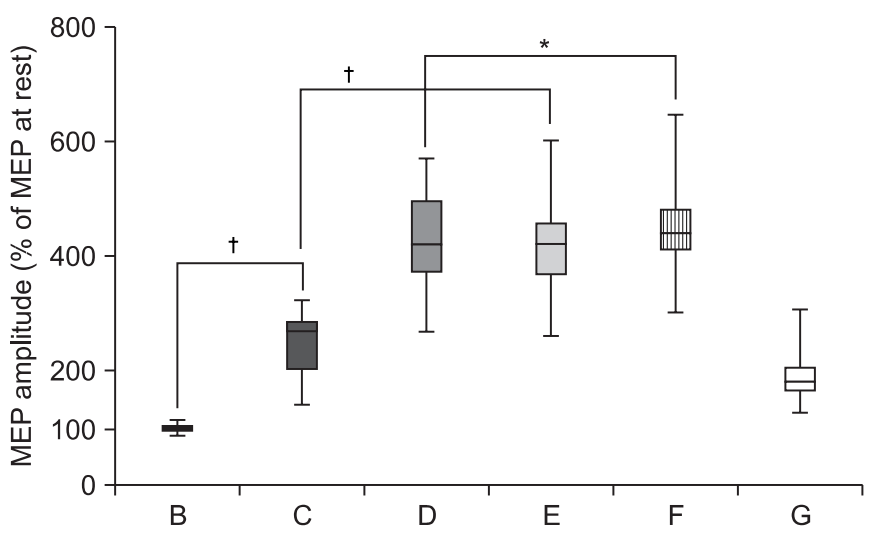

Fig. 2. Increment of percentage MEP values in healthy subjects. Mean $( \pm$ SEM) values of the MEP amplitude measured during the experiment, expressed as percentage of the MEP at rest in 30 healthy subjects. Based on repeated measures ANOVA, the percentage MEP amplitude of observation and imagery of the activity of the hand of another individual with a mirror (E) was significantly increased compared with observation and imagery of the activity of the hand of another individual without a mirror (C) ( $\left.{ }^{\dagger} \mathrm{p}<0.01\right)$. In addition, the percentage MEP amplitude of observation and imagery of self hand activity with a mirror (F) was significantly increased compared with observation and imagery of own hand activity without a mirror (D) ( $\left.{ }^{*} \mathrm{p}<0.05\right)$. (A) rest; (B) imagery; (C) observation and imagery of the activity of the hand of another individual without a mirror; (D) observation and imagery of self hand activity without a mirror; (E) observation and imagery of the activity of the hand of another individual with a mirror; (F) observation and imagery of own symmetric hand activity with a mirror; and $(G)$ observation and imagery of own asymmetric hand activity with a mirror.
(Fig. 2).

Comparing MEP latency across the seven testing conditions shows a significant decrement $\left(\mathrm{F}_{6,162}=290.1\right)$ $(p<0.001)$ (Fig. 3). The mean MEP latency was significantly lower in (B) imagery than in (A) relaxation state $(\mathrm{p}<0.001)$ (Fig. 3). The mean MEP latency was significantly lower in (C) observation and imagery than in (A) relaxation state or (B) imagery only $(\mathrm{p}<0.001)$ (Fig. 3$)$. The comparison of the condition (C) and (D) revealed that the mean MEP latency of (D) observation and imagery of own hand activity was lower than that of (C) observation and imagery of the activity of the hand of another individual $(p<0.001)$. The analysis of mirror conditions showed that the mean MEP latency was significantly lower in the mirror condition (E) observation and imagery of the activity of the hand of another individual with a mirror than in (C) observation and imagery of the activity of the hand of another individual without a mirror $(p<0.001)$

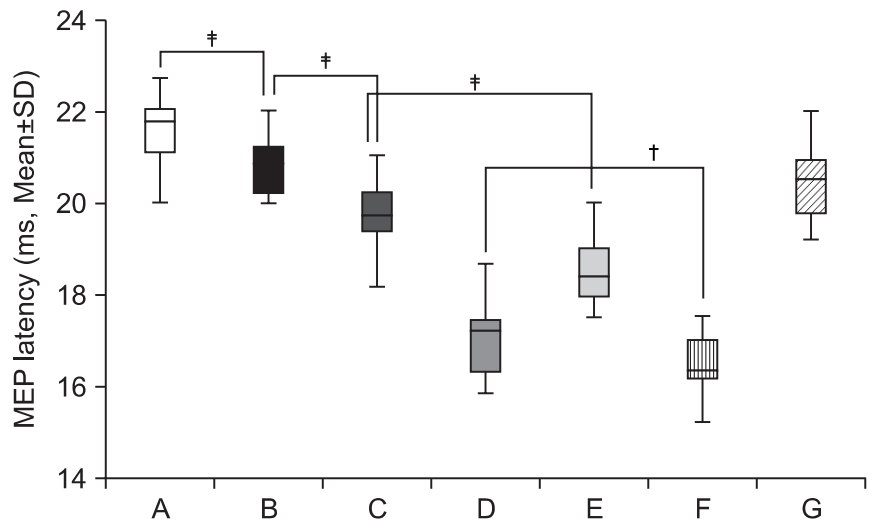

Fig. 3. Decrement of MEP latency values in healthy subjects. Mean $( \pm$ SEM $)$ values of the MEP latency during the experiment in 30 healthy subjects. Based on repeated measures ANOVA, the MEP latency of observation and imagery of the activity of the hand of another individual with a mirror (E) was significantly decreased compared with observation and imagery of the activity of the hand of another individual without a mirror (C) $\left({ }^{\ddagger} \mathrm{p}<0.001\right)$. In addition, the MEP latency of observation and imagery of own hand activity with a mirror (F) was significantly decreased compared with observation and imagery of own hand activity without a mirror (D) ( $\left.{ }^{\dagger} \mathrm{p}<0.01\right)$. (A) rest; (B) imagery; (C) observation and imagery of the activity of the hand of another individual without a mirror; (D) observation and imagery of own hand activity without a mirror; (E) observation and imagery of the activity of the hand of another individual with a mirror; (F) observation and imagery of own symmetric hand activity with a mirror; and $(G)$ observation and imagery of own asymmetric hand activity with a mirror. 
(Fig. 3). The test condition of (F) observation and imagery of own hand activity with a mirror yielded lower MEP latency than (D) observation and imagery of own hand activity without a mirror $(\mathrm{p}<0.01)$ (Fig. 3 ).

Interestingly, the mirror condition (F) observation and imagery of own ipsilateral symmetric hand activity with a mirror yielded a higher percentage of MEP amplitude and lower latency than (G) observation and imagery of own ipsilateral asymmetric hand activity with a mirror $(\mathrm{p}<0.001)$ (Fig. 4).

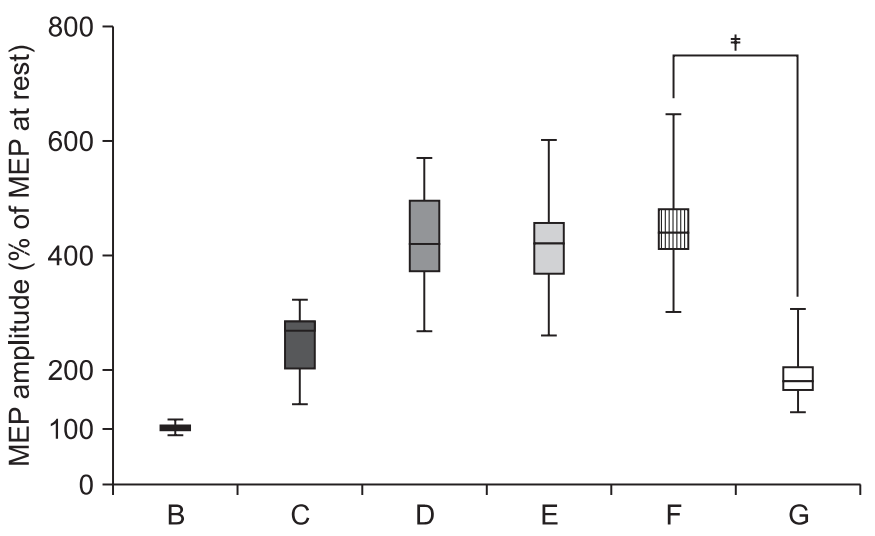

Facilitation of corticospinal excitability during motor imagery and mirror therapy in stroke patients

The comparison of percantage MEP amplitudes in 30 stroke patients across the four testing conditions revealed a pattern of significant differences during experiment $\left(\mathrm{F}_{3,87}=34.0\right)(\mathrm{p}<0.001)$ (Fig. 5, Table 3). The percentage of MEP amplitude increment and latency decrement were significantly greater in (E) observation and imagery of the activity of the hand of another individual with a mirror than in (C) observation and imagery of the activity of the hand of another individual without a mirror $(p<0.001)$.

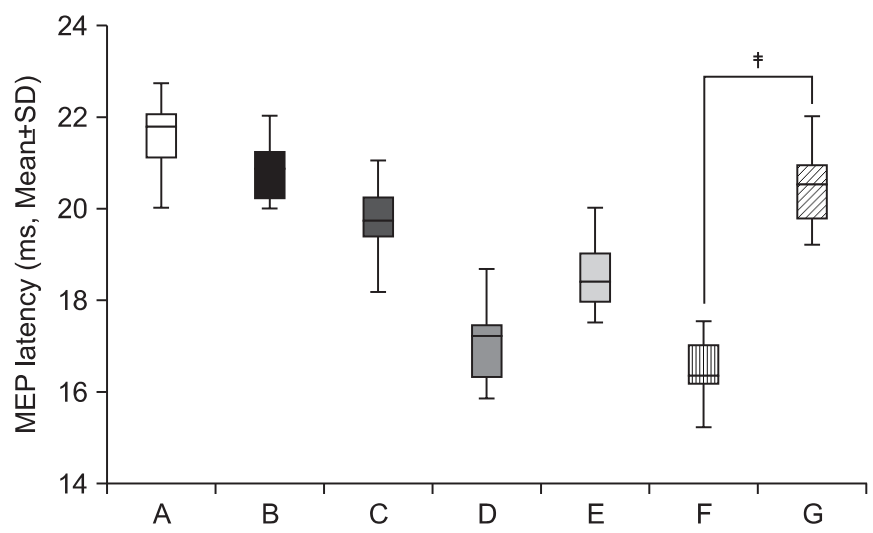

Fig. 4. Observation-execution matching. The percentage MEP amplitude increment and latency decrement of observation and imagery of own symmetric hand activity with a mirror (F) was significantly increased compared with observation and imagery of own asymmetric hand activity with a mirror $(\mathrm{G})\left({ }^{\ddagger} \mathrm{p}<0.001\right)$ in 30 healthy subjects (observation-execution matching).
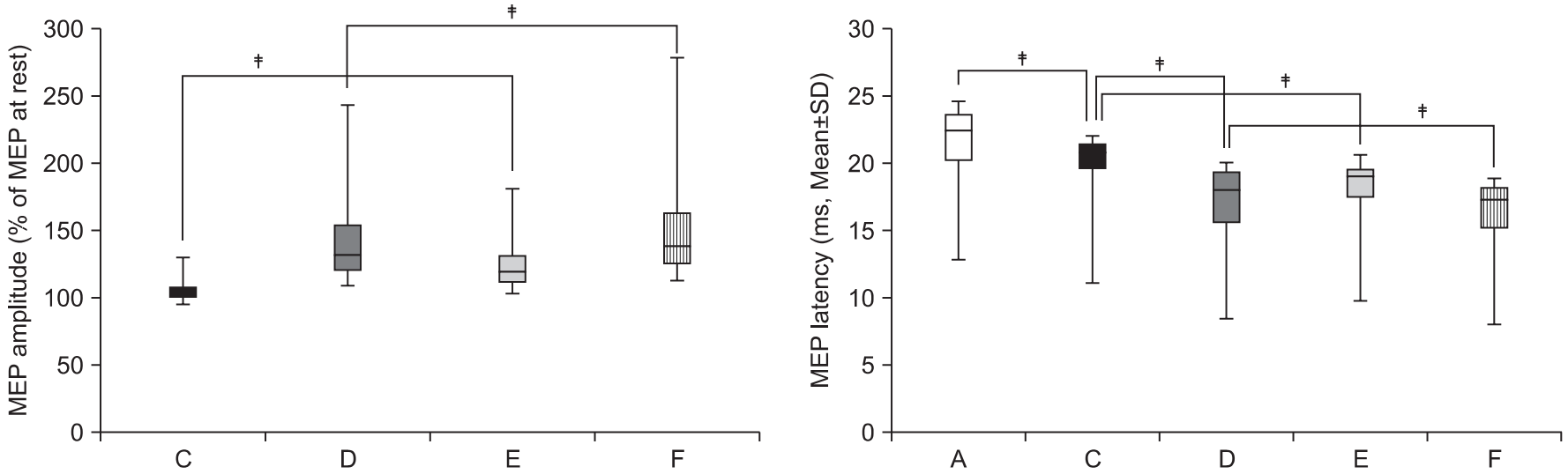

Fig. 5. Increment of percentage MEP amplitude and decrement of MEP latency in stroke patients. The percentage MEP amplitude increment and latency decrement of MEPs were significantly greater in the activity of the hand of another individual with a mirror (E) than in observation and imagery of the activity of the hand of another individual without a mirror (C) $\left({ }^{\ddagger} \mathrm{p}\right.$ $<0.001)$. In addition, the percentage MEP amplitude increment and latency decrement of MEPs were significantly greater in own hand activity with a mirror (F) than in observation and imagery of own hand activity without a mirror (D) $\left({ }^{+} \mathrm{p}<0.01,{ }^{*} \mathrm{p}\right.$ $<0.001$ ). (A) rest; (C) observation and imagery of the activity of the hand of another individual without a mirror; (D) observation and imagery of self hand activity without a mirror; (E) observation and imagery of the activity of the hand of another individual with a mirror; (F) observation and imagery of the own hand activity with a mirror. 
In addition, the percentage of MEP amplitude increment and latency decrement were significantly greater in (F) observation and imagery of own hand activity with a mirror than in (D) observation and imagery of own hand activity without a mirror $(\mathrm{p}<0.01$ and $\mathrm{p}<0.001$, respectively) (Fig. 5).

Further, mean MEP latencies across the seven testing conditions showed a significant differences $\left(\mathrm{F}_{4,120}=\right.$ 258.8) ( $\mathrm{p}<0.001)$ (Fig. 5). The mean MEP latency was significantly lower in (C) observation and imagery than in (A) relaxation state $(\mathrm{p}<0.001)$ (Fig. 3$)$.

\section{DISCUSSION}

According to recent TMS and fMRI research, the brain function of the ipsilesional side decreases during the recovery after stroke. ${ }^{18,24}$ Abnormal strong interhemispheric inhibition from the contralesional hemisphere was observed in stroke survivors, which is associated with poor functional outcome and ipsilesional cortical activation and is important for good motor recovery. ${ }^{18}$ This indicates that ipsilesional motor cortical priming after a stroke may be considered an important part of the management of the balance between hemispheres and of the recovery of functions. The method of voluntary exercise evokes the strongest facilitation at the cortex and spine levels. However, an alternative facilitation method is necessary because voluntary movements are difficult for stroke patients with severe paralysis of affected limbs.

In this experiment, both healthy subjects and stroke patients exhibited motor corticospinal facilitation in conditions of motor imagery and observation, voluntary movement, indirect observation using a mirror, and voluntary movement using a mirror. Previous study revealed that age does not seem to be a significant contributor to variations in MEP amplitude ${ }^{25,26}$ so, did not match the mean age of the two groups.

Facilitation of corticospinal excitability during observation and motor imagery

This research was meaningful because corticospinal facilitation induced by motor imagery with or without observation was found in stroke patients, although this type of facilitation is frequently reported in healthy subjects. Clark et al. showed that a stronger facilitation effect was observed for imagination of the movements compared with observation of the movements, and that a higher amplitude of MEP was found in mimicking the movements compared with imagining the movements. ${ }^{27}$ Further, another report showed that kinesthetic imagination of the movements yielded stronger MEP amplitude than visual imagination of the movements. ${ }^{10}$ The mechanism through which motor imagery facilitates the motor cortex is based on the activation of the mirror neuron system. ${ }^{28}$

The mirror neuron system is associated with the extended motor network of the cerebral cortex, which is involved in the execution and control of movements. According to recent extended motor network theory, the ipsilesional prefrontal cortex, the dorsal premotor area, and the primary motor cortex form a network with the premotor area, the prefrontal cortex, the parietal lobe, and the cerebellum in the contralesional hemisphere. ${ }^{7}$ Sharma et al. used fMRI to show that there are no differences in the regional activation patterns of the extended motor network in either healthy individuals or stroke patients. ${ }^{7}$ In addition, execution of movement yielded stronger activation of the extended motor network compared with imagination of movement in healthy individuals, and in stroke patients. ${ }^{7}$ These fMRI results and similar activation patterns in healthy individuals and stroke patients are consistent with our results.

Facilitation of corticospinal excitability during mirror therapy

The hypothesis that the mirror-reflected image of movements of the unaffected limb evokes a visual illusion and activates the ipsilesional cortex had not previously been confirmed. ${ }^{14,15}$ In this study, we confirmed this hypothesis using TMS. Greater facilitation of the ipsilesional motor cortex was evoked by indirect observation of hand activity reflected by a mirror compared with that obtained using direct observation of hand activities. This facilitation may be associated with the activation of the ipsilesional hemisphere via a visual illusion evoked by the mirror, as well as the activation of the mirror neuron system. In similar fMRI experiments conducted by Merians et al. using three healthy subjects, the contralateral motor cortex was activated by the visual illusion evoked by a virtual mirror program. ${ }^{29}$ In addition, the fMRI study performed by Sinoura et al. yielded experimental results 
that movements performed looking at a mirror activated the mirror neuron system. ${ }^{30}$ Funase et al., however, reported an absence of differences in MEP amplitude using TMS between indirect observation using a mirror and direct observation. ${ }^{21}$ These inconsistencies may result from the different methods used and the depth of subjects' imagination or observation, the vividness of imagination, or the complexity of the movements and tasks.

In this study, the rates of amplitude increment and latency decrement of MEPs were significantly greater during the observation of the activity of the hand of another individual with a mirror than without a mirror. Furthermore, the rates of amplitude increment and latency decrement were significantly greater in the observation of their own symmetric ipsilateral hand activity with a mirror than the observation without a mirror, and in both the healthy control and stroke patient groups. This could represent evidence of a neural mechanism that supports the contention that mirror therapy should be provided simultaneously with movement observation and voluntary exercise during stroke rehabilitation.

One of the interesting points of this study was that the facilitation of MEP was only observed in the muscles seen through the mirror. This is in agreement with research suggesting the existence of observation-execution matching, as observation of movement resulted in selective activation of the motor cortex in the muscle involved in that movement. ${ }^{31,32}$

An insufficient number of subjects and the heterogeneity of the lesions, onset time, and functional level may represent the limitations of our study. Further, it is hard to generalize this result to the total group of stroke patients, because subjects with good functional ability were recruited. The lack of an assessment of the imaginative and attention-based abilities of the subjects is another drawback of this study. In future investigations, clinical studies examining the effectiveness of the mirror therapy program according to various stroke subgroups, training method, and training duration should also be considered.

\section{CONCLUSION}

In both groups, corticospinal excitability was facilitated by viewing a mirror image of ipsilateral hand activity. This facilitation provided by mirror therapy was highly selective for the viewing of the muscles involved in the task. This may represent neurophysiological evidence that supports the application of a mirror therapy paradigm to the rehabilitation of upper extremities in stroke patients.

\section{ACKNOWLEDGEMENTS}

Thanks to Sangwoo Cho at the Department of Biomedical Engineering, Hanyang University and Hae Sun Lee at the Department of Medical-Statistics in Yonsei University for the valuable comments on the statistical analysis of obtained results and special thanks to Miae Jung, Dal-Jae Im and Yon Joon Kim at the Eulji Hospital for helping with the experiments.

This research was supported by the Basic Science Research Program through the National Research Foundation of Korea (NRF) and funded by Korea Government (MEST) (No. 2011-0002451).

\section{REFERENCES}

1. Han CE, Arbib MA, Schweighofer N. Stroke rehabilitation reaches a threshold. PLoS Comput Biol 2008; 4: e1000133

2. Page SJ, Sisto SA, Levine P. Modified constraint-induced therapy in chronic stroke. Am J Phys Med Rehabil 2002; 81: 870-875

3. Nudo RJ. Adaptive plasticity in motor cortex: implications for rehabilitation after brain injury. J Rehabil Med 2003; 41 Suppl: 7-10

4. Krakauer JW. Motor learning: its relevance to stroke recovery and neurorehabilitation. Curr Opin Neurol 2006; 19: 84-90

5. Oujamaa L, Relave I, Froger J, Mottet D, Pelissier JY. Rehabilitation of arm function after stroke. Literature review. Ann PhysRehabil Med 2009; 52: 269-293

6. Mulder T. Motor imagery and action observation: cognitive tools for rehabilitation. J Neural Transm 2007; 114: 1265-1278

7. Sharma N, Baron JC, Rowe JB. Motor imagery after stroke: relating outcome to motor network connectivity. Ann Neurol 2009; 66: 604-616

8. Kuhtz-Buschbeck JP, Mahnkopf C, Holzknecht C, Siebner H, Ulmer S, Jansen O. Effector-independent 
representations of simple and complex imagined finger movements: a combined fMRI and TMS study. Eur J Neurosci 2003; 18: 3375-3387

9. Ruby P, Decety J. What you believe versus what you think they believe: a neuroimaging study of conceptual perspective-taking. Eur J Neurosci 2003; 17: 2475-2480

10. Stinear CM, Byblow WD, Steyvers M, Levin O, Swinnen SP. Kinesthetic, but not visual, motor imagery modulates corticomotor excitability. Exp Brain Res 2006; 168: 157-164

11. Page SJ, Levine $\mathrm{P}$, Leonard A. Mental practice in chronic stroke: results of a randomized, placebocontrolled trial. Stroke 2007; 38: 1293-1297

12. Verbunt JA, Seelen HA, Ramos FP, Michielsen BH, Wetzelaer WL, Moennekens M. Mental practicebased rehabilitation training to improve arm function and daily activity performance in stroke patients: a randomized clinical trial. BMC Neurol 2008; 8: 7

13. Ramachandran VS, Rogers-Ramachandran D. Synaesthesia in phantom limbs induced with mirrors. Proc Biol Sci 1996; 263: 377-386

14. Dohle C, Pullen J, Nakaten A, Kust J, Rietz C, Karbe H. Mirror therapy promotes recovery from severe hemiparesis: a randomized controlled trial. Neurorehabil Neural Repair 2009; 23: 209-217

15. Yavuzer G, Selles R, Sezer N, Sütbeyaz S, Bussmann JB, Köseoğlu F, Atay MB, Stam HJ. Mirror therapy improves hand function in subacute stroke: a randomized controlled trial. Arch Phys Med Rehabil 2008; 89: 393-398

16. Liepert J, Hamzei F, Weiller C. Motor cortex disin hibition of the unaffected hemisphere after acute stroke. Muscle Nerve 2000; 23: 1761-1763

17. Shimizu T, Hosaki A, Hino T, Sato M, Komori T, Hirai S, Rossini PM. Motor cortical disinhibition in the unaffected hemisphere after unilateral cortical stroke. Brain 2002; 125: 1896-1907

18. Calautti C, Naccarato M, Jones PS, Sharma N, Day DD, Carpenter AT, Bullmore ET, Warburton EA, Baron JC. The relationship between motor deficit and hemisphere activation balance after stroke: a 3T fMRI study. Neuroimage 2007; 34: 322-331

19. Stinear CM, Barber PA, Coxon JP, Fleming MK, Byblow WD. Priming the motor system enhances the effects of upper limb therapy in chronic stroke. Brain 2008; 131:

\section{1-1390}

20. Garry MI, Loftus A, Summers JJ. Mirror, mirror on the wall: viewing a mirror reflection of unilateral hand movements facilitates ipsilateral M1 excitability. Exp Brain Res 2005; 163: 118-122

21. Funase K, Tabira T, Higashi T, Liang N, Kasai T. Increased corticospinal excitability during direct observation of self movement and indirect observation with a mirror box. Neurosci Lett 2007; 419: 108-112

22. Hallett M. Transcranial magnetic stimulation and the human brain. Nature 2000; 406: 147-150

23. Rossini PM, Barker AT, Berardelli A, Caramia MD, Caruso G, Cracco RQ, Dimitrijevic MR, Hallett M, Katayama Y, Lucking CH, et al. Non-invasive electrical and magnetic stimulation of the brain, spinal cord and roots: basic principles and procedures for routine clinical application. Report of an IFCN committee. Electroencephalogr Clin Neurophysiol 1994; 91: 79-92

24. Talelli P, Greenwood RJ, Rothwell JC. Arm function after stroke: neurophysiological correlates and recovery mechanisms assessed by transcranial magnetic stimulation. Clin Neurophysiol 2006; 117: 1641-1659

25. Wassermann EM. Variation in the response to transcranial magnetic brain stimulation in the general population. Clin Neurophysiol 2002; 113: 1165-1171

26. Leonard G, Tremblay F. Corticomotor facilitation associated with observation, imagery and imitation of hand actions: a comparative study in young and old adults. Exp Brain Res 2007;177:167-175

27. Clark S, Tremblay F, Ste-Marie D. Differential modulation of corticospinal excitability during observation, mental imagery and imitation of hand actions. Neuropsychologia 2004; 42: 105-112

28. Buccino G, Solodkin A, Small SL. Functions of the mirror neuron system: implications for neurorehabilitation. Cogn Behav Neurol 2006; 19: 55-63

29. Merians AS, Tunik E, Fluet GG, Qiu Q, Adamovich SV. Innovative approaches to the rehabilitation of upper extremity hemiparesis using virtual environments. Eur J Phys Rehabil Med 2009; 45: 123-133

30. Shinoura N, Suzuki Y, Watanabe Y, Yamada R, Tabei Y, Saito K, Yagi K. Mirror therapy activates outside of cerebellum and ipsilateral M1. NeuroRehabilitation 2008; 23: 245-252

31. Sundara M, Namasivayam AK, Chen R. Observationexecution matching system for speech: a magnetic 
Youn Joo Kang, et al.

stimulation study. Neuroreport 2001; 12: 1341-1344

32. Sakamoto M, Muraoka T, Mizuguchi N, Kanosue K.

Execution-dependent modulation of corticospinal excitability during action observation. Exp Brain Res 2009; 199: 17-25 